\title{
INTELLECTUAL MULTI-LEVEL SYSTEM FOR NEURO-FUZZY AND COGNITIVE ANALYSIS AND FORECAST OF SCIENTIFIC- TECHNOLOGICAL DEVELOPMENT AND INNOVATIVE
}

\author{
Sergey Gorbachev ${ }^{\text {a }}$ \\ National Research Tomsk State University, 634050 Tomsk, Russia
}

\begin{abstract}
The article is devoted to description of structure and operation algorithms of a multilevel intellectual system for analysis and forecast of scientific-technological and innovative development of objects and territories based on expert methods, trainable models of fuzzy logic and fuzzy cognitive analysis. The article analyses distinctions and advantages of the system in making forecast evaluation and scenarios of the dynamics of multidimensional feature objects and processes. The authors have built a neutrosophic cognitive map for interaction of technologies based on the results of foresight studies.
\end{abstract}

\section{Introduction}

The current state of research in the field of dynamics of scientific and technological progress is closely connected with the methods of monitoring and analysing the emergence of a new technological order. For example, in [1] S.Glaziev gives a characterization of a new way as a certain set of conjugate industries located approximately at the same stage of technical development. In a long-term perspective, scientific and technological progress will be carried out through renovation of the traditional technological order. Therefore, when creating a model of modern technical and economic processes, researchers meet the problem of blurriness and uncertainty of the factors of technological change [2].

Global modelling of these processes began in the 1970s in the works of J. Forrester and D.Meadows [3], in which technological development and resource constraints were taken into account. These studies had great influence on the work of other researchers and public opinion and consequently the ideas of sustainable (self-sustaining) development gained significance.

Based on the emerging issues of the adequacy of theoretical models, optimization and imitation groups were developed to formalize socio-economic and scientific-technological processes.

a Corresponding author: spp03@sibmail.com 
An optimization model is characterized by the non-closure of the equation systems describing them; it means that some of the variables corresponding to the control actions are not specified.

In this case, investigation of objects consists in finding the values of these variables, which optimize a certain functional as an achievement of the specific goal set beforehand.

Among the simulation models, first, it is worth noting the models of Forrester, Meadows and Mesarovic-Pestel. They are a system of nonlinear differential equations. Using this approach, a model is provided with a pre-selected and fixed control mechanism, which in fact is a decision-making model built on the system development background. In this case, the research mechanism is associated with a search of various "policies" and an analysis of their influence on the system behaviour. By "politicians", we mean various combinations of hypotheses about functional relationships, structure of the control mechanism, and numerical values of the parameters. This helps to understand the dynamics of the processes under study and to identify possible critical development trends. At the same time, the fixation of the control mechanism does not presuppose a conscious change of the existing development tendencies by a person and does not allow analysing all the possibilities of the system with admissible control actions.

The purpose of this study is to develop global models and use them in studying various scenarios based on the development of a multilevel intellectual system for analysing and predicting the dynamics of socio-economic, technical-economic and scientifictechnological processes. A set of mathematical, intellectual, expert, and cognitive methods with logical interpretation of the research results can form the core of this system. For the first time in the world science, the authors of the article developed and implemented a neuro-fuzzy model of the trajectory of world technical and economic development, which allows measuring the level and rates of world technical and economic development and calculating the parameters of countries relative to the standard [4]. The development of these studies was the development of hybrid methods for training fuzzy models based on the synthesis of trained fuzzy decision trees with the conclusion of diagnostic decision rules that allow solving the problem of multicriteria classification of scientific and technological directions and technologies by the target factor.

In our opinion, foresight methods represent an effective analytical tool for identifying priority areas of science, technology and innovation based on expert assessments of strategies and prospects for innovative development, identifying technological breakthroughs that may have an impact on society and the economy in the medium and long term [5].

\section{Structural and functional organization of the intellectual system for analysis and forecasting}

Foresight methods allow creating an adequate system for monitoring, analysing and forecasting the development of science and technology. Thus, a multilevel and nonlinear system of scientific priorities and innovative technologies can be presented as a hierarchically constructed system with such specific features as the principles of adaptation to the existing social and economic situation of global and national scale, and evolution over time taking into account the trends of the world technical and technological development.

Based on the results of the study, a structural-functional scheme of an intellectual system for analysis and forecast of scientific and technological development was developed. The system includes three levels linked together by analysis and forecast (Figure 1). 


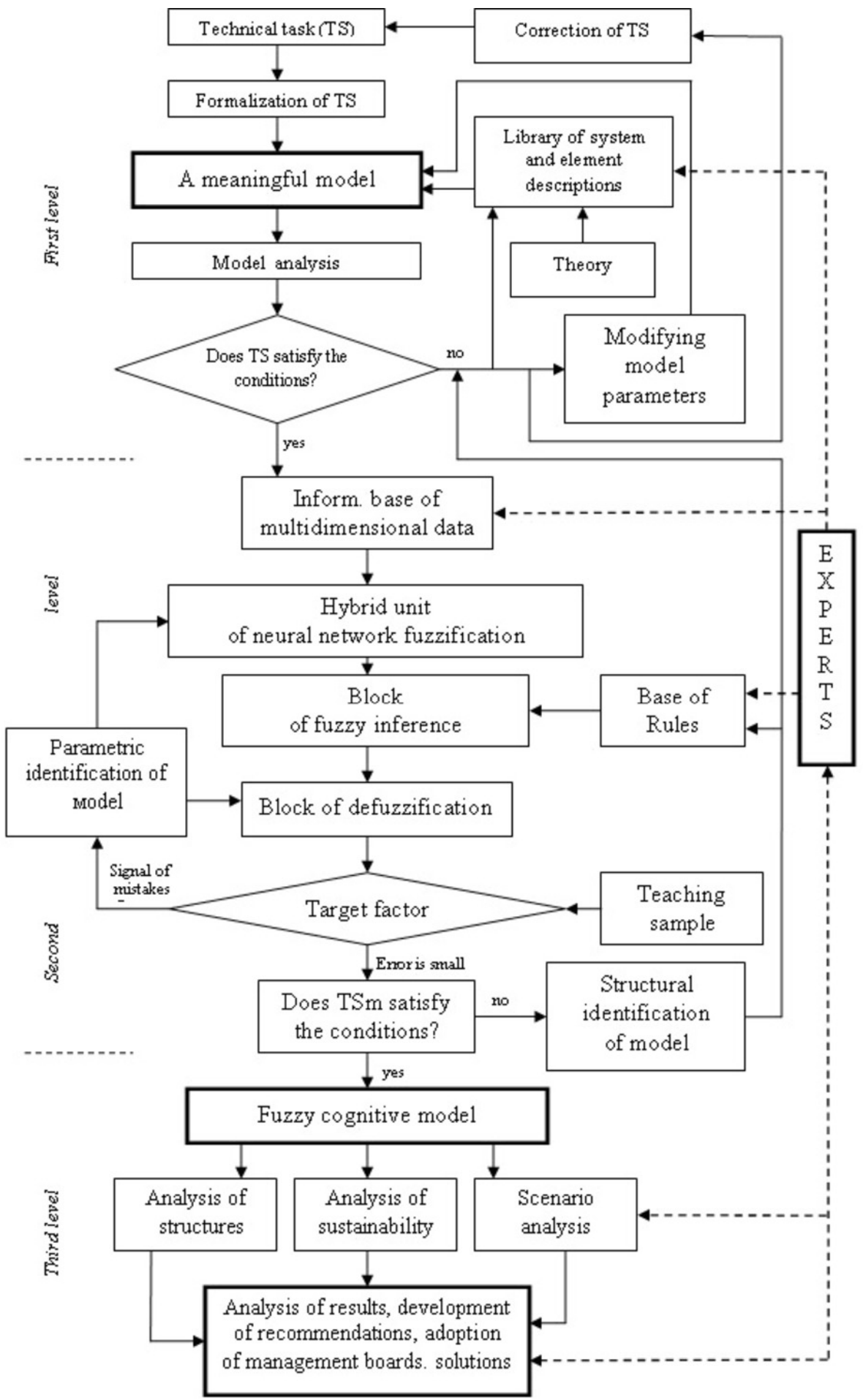

Fig. 1. Structural-functional scheme of intellectual multi-level system for analysis and forecast of scientific, technological and innovative development. 


\subsection{A meaningful model (level 1)}

At the first level, we developed a conceptual methodology for forming a meaningful research model depending on the target factor based on statistical and other information that can be quantitative or qualitative. At the same time, we studied the methodological problems of forming the pool of experts taking into account their professional identity as well as the issues of qualitative representation of experts' knowledge during the surveys [6]. During the expert seminars, we examined the scope, limitations and assumptions, as well as the principles for formation and selection of indexes and indicators for the expert evaluation of the effectiveness of scientific and technological directions and technologies. During the examination process, we considered not only the features and requirements of innovative development of the Russian economy, its technological, production, scientific and personnel potential, but also the trajectory of world technical and economic development.

At the initial stage, the content model is characterized by a set of quantitative and qualitative characteristics that can be identified by experts. In the future, it can be replenished with other indicators, depending on social-economic, political, and other target factors.

\subsection{Multidimensional adaptive model of neuro-fuzzy inference (level 2)}

The second level is connected with formation of the information base of the characteristics by the results of foresight research, solution of computational analytical problems with the conclusion of fuzzy diagnostic decision rules. The construction of classical adaptive neuro fuzzy inference systems (ANFIS) is associated with formalization of input parameters and target indicators of the effectiveness of scientific (innovative) projects and technologies in the form of a vector of interval values (fuzzy interval); the hit in each interval of this vector is characterized by some degree of uncertainty [7]. This procedure is called "fuzzification". Based on the initial information, experience and intuition, experts often and confidently can make quantitative evaluation of the boundaries (intervals) of possible (permissible) values of parameters and the range of their most possible (preferred) values. For example, experts can characterize the primary data using triangular fuzzy numbers with the following membership function $\mu_{A}(x)$ of the fuzzy set $A$ :

$$
\mu_{A}(x)=\left\{\begin{array}{l}
0, \text { if } x \leq a_{\min } \\
\frac{x-a_{\min }}{c-a_{\min }}, \text { if } a_{\min }<x<c \\
\frac{a_{\max }-x}{a_{\max }-c}, \text { if } c \leq x<a_{\max } \\
0, \text { if } x \geq a_{\max }
\end{array}\right.
$$

This expression simulates the following expert position: "parameter $A$ is approximately equal to $c$ and uniquely enters to the segment $\left[a_{\min }, a_{\max }\right]$ ".

In practice, an analytical representation of the membership function is often used [5], for example, the Gaussian membership function has the form:

$$
\mu_{A}(x)=e^{-\frac{(x-c)^{2}}{2 \sigma^{2}}}
$$

where $c$ is a center and $\sigma$ is a standard deviation (width).

Special algorithms for the fazzification of expert data have been developed [8], which are described not in numerical but in a rank (ordinal) scale. For example, it can be 
described as a sequence of ordered linguistic variables $x_{1}, \ldots, x_{n}$, where one of the ranks $t$ $\left(t=1, \ldots, t_{k}\right.$, for example, low, below average, medium, above average, and high) can be assigned to each of the observations $x_{k}$.

For the automatic formation of a rule base, a classification fuzzy decision tree based on a set of characteristics has been developed; it can be viewed as a hierarchical network with direct signal propagation [9]. Therefore, to adapt its parameters (centers and width of membership functions, as well as parameters of leaf identification), it is possible to use a hybrid algorithm for back propagation of the error by reversing each leaf node from the root node of the tree. Figure 1 presents a scheme of the parametric identification of a model. This procedure allows us to synthesize a decision tree with a small number of elements, which has a high level of generalization, with the possibility of a linguistic interpretation of the result by deriving diagnostic decision rules. Because of the optimized network scheme and the reduced number of calculations, the developed hybrid adaptive neural-fuzzy algorithms, which implement the method of classification and fine-tuning the decision tree parameters, increase the speed of the classical algorithm and preserve the hierarchical structure of the tree without any damage to interpretation.

In addition to the classification problem, the problem of multidimensional approximation with a logical conclusion that implements the mapping $F: \mathfrak{R}^{n} \rightarrow \mathfrak{R}$ is of interest. Further, we will show the possibility of solving this problem based on constructing a neural-fuzzy approximating model and fine-tuning the parameters of the rule consequents.

\subsection{A fuzzy cognitive model (level 3)}

The third level is associated with fuzzy cognitive modeling, which solves a number of forecasting problems [10]. Currently, one of the developing areas of cognitive sciences is cognitive modeling, which is an interdisciplinary scientific direction that unites the theory of cognition, cognitive psychology, neurophysiology, cognitive linguistics and a theory of artificial intelligence. The results of such synthesis, for example, in the form of the intellectual decision support system described, have a pronounced synergistic effect.

Traditionally, the cognitive model is a cognitive map [11] in the form of a signoriented graph (digraph) $G=<V, E>$, where:

$V$ is a set of concepts (vertices),

$V_{i} \in V, i=1,2, \ldots, k$ are the elements of the study system;

$E$ is a set of arcs, and the $\operatorname{arcs} e_{i j} \in E, i, j=1,2, \ldots, N$ reflect the relationship between the concepts.

The cognitive map allows analyzing both qualitative and quantitative data, and the overall goal of cognitive modeling is to generate and test hypotheses about the functional structure of the observed situation. Iterations are conducted up to a certain state of causeeffect relations, the structural scheme of which will enable us to understand and analyze behavior of the system.

\section{Results and Discussion}

Based on the conducted foresight research, experts evaluated the degree of interaction of innovative technologies in conditions of transition to the sixth technological order from the point of view of influence on the process of conjugation and formation of the load-bearing sectors and clusters of economy of a new technological order. 
Figure 2 shows a neutrosophic cognitive map (NCM) of the technologies interactions generated based on the results of foresight studies (numbering corresponds to their technology list in the questionnaire).

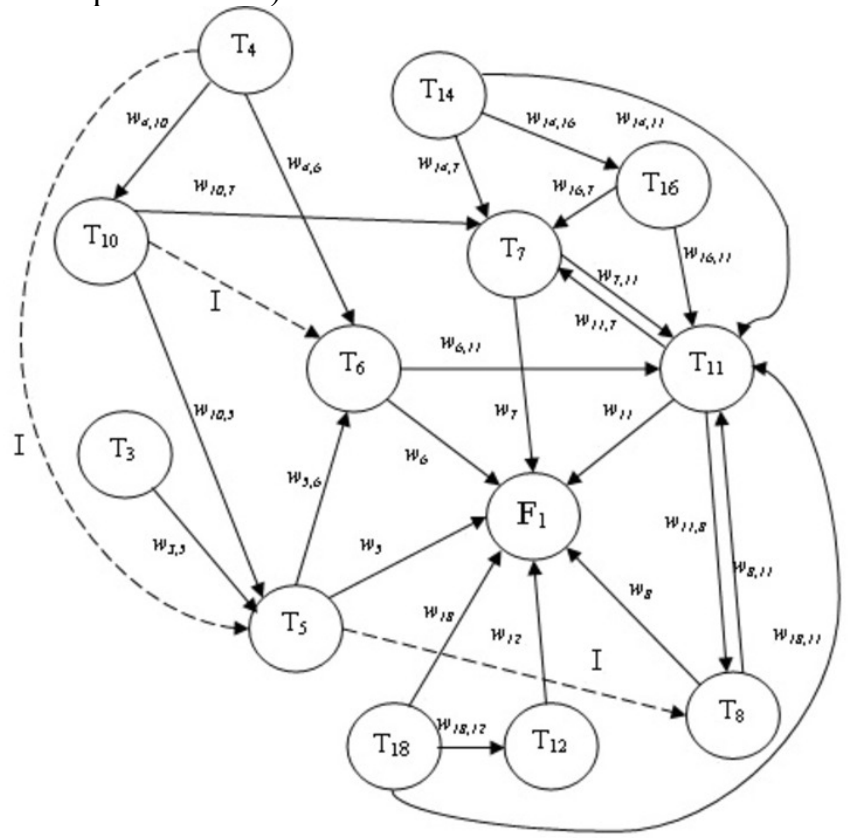

Fig. 2. Neutrosophic cognitive map of the conjugation technologies. form:

The adjacency matrix obtained from the Neutrosophic cognitive map has the following

$\begin{array}{ccccccccccccc}0 & 0 & w_{3,5} & 0 & 0 & 0 & 0 & 0 & 0 & 0 & 0 & 0 & 0 \\ 0 & 0 & I & w_{4,6} & 0 & 0 & w_{4,10} & 0 & 0 & 0 & 0 & 0 & 0 \\ 0 & 0 & 0 & w_{5,6} & 0 & I & 0 & 0 & 0 & 0 & 0 & 0 & w_{5} \\ 0 & 0 & 0 & 0 & 0 & 0 & 0 & w_{6,11} & 0 & 0 & 0 & 0 & w_{6} \\ 0 & 0 & 0 & 0 & 0 & 0 & 0 & w_{7,11} & 0 & 0 & 0 & 0 & w_{7} \\ 0 & 0 & 0 & 0 & 0 & 0 & 0 & w_{8,11} & 0 & 0 & 0 & 0 & w_{8} \\ 0 & 0 & w_{10,5} & I & w_{10,7} & 0 & 0 & 0 & 0 & 0 & 0 & 0 & 0 \\ 0 & 0 & 0 & 0 & w_{11,7} & w_{11,8} & 0 & 0 & 0 & 0 & 0 & 0 & w_{11} \\ 0 & 0 & 0 & 0 & 0 & 0 & 0 & 0 & 0 & 0 & 0 & 0 & w_{12} \\ 0 & 0 & 0 & 0 & w_{14,7} & 0 & 0 & w_{14,11} & 0 & 0 & w_{14,16} & 0 & 0 \\ 0 & 0 & 0 & 0 & w_{16,7} & 0 & 0 & w_{16,11} & 0 & 0 & 0 & 0 & 0 \\ 0 & 0 & 0 & 0 & 0 & 0 & 0 & w_{18,11} & w_{18,12} & 0 & 0 & 0 & w_{18} \\ 0 & 0 & 0 & 0 & 0 & 0 & 0 & 0 & 0 & 0 & 0 & 0 & 0\end{array}$

The purpose of the analysis is to calculate the degree of exposure to one or more of the concepts (technologies) taking into account their condition on the target by meansof NCM . 
Let us consider the impact the concept of the $T_{5}$, on the system, which corresponds to the respective genomic, proteomes and post-genomic technologies. Based on definition 5 , we show how to find is a fixed point under the condition that the system is affected by

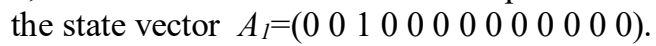

First, we multiply the vector $A_{l}$ by the neutrosophic adjacency matrix $W$, and build a new state vector in accordance with the result. Then we multiply the vector $A_{2}$ obtained on the previous stage by the neutrosophic adjacency matrix $W$, pre-adding a unity to the third component of the vector (this is necessary in order to consider the initial single exposure). Further, in a similar way, we write a state vector to the result of multiplication obtained. Iterative procedure is conducted until the state vector at $n$ step will be equal to the state vector at $n-1$ step. This means that we managed to reach the fixed point as a result of the iterative procedure:

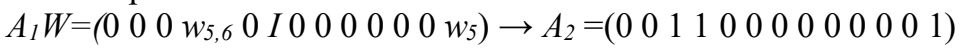

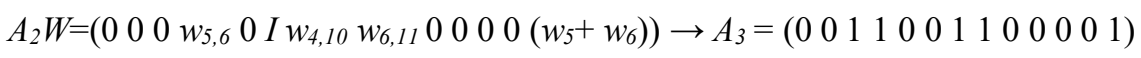

$$
\begin{aligned}
& A_{3} W=\left(00 w_{10,5}\left(w_{5,6}+I\right)\left(w_{10,75}+w_{11,7}\right)\left(I+w_{11,8}\right) 0 w_{6,11} 00000\left(w_{5}+w_{6}+w_{11}\right)\right) \rightarrow \\
& A_{4}=\left(\begin{array}{lllllllllllll}
0 & 0 & 1 & 1 & 1 & 1 & 0 & 1 & 0 & 0 & 0 & 0 & 1
\end{array}\right)
\end{aligned}
$$

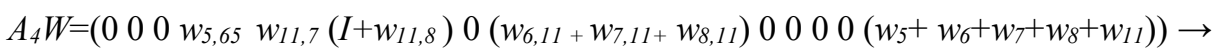

$$
\begin{aligned}
& A_{5}=\left(\begin{array}{lllllllllllll}
0 & 0 & 1 & 1 & 1 & 1 & 0 & 1 & 0 & 0 & 0 & 0 & 1
\end{array}\right)=A_{4}
\end{aligned}
$$

In this case, we managed to reach the fixed point in 4 iterations. The value $A_{4} W$ represents the system state vector, each element of which describes the impact applied to the corresponding concept in the result of a single impact $T_{5}$.

Let us consider a special case of the simultaneous action of several concepts $T_{5}, T_{7}, T_{8}$,

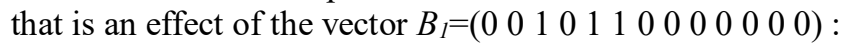

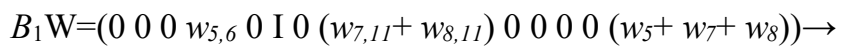

$$
\begin{aligned}
& B_{2}=\left(\begin{array}{lllllllllllll}
0 & 0 & 1 & 1 & 1 & 1 & 0 & 1 & 0 & 0 & 0 & 0 & 1
\end{array}\right)
\end{aligned}
$$

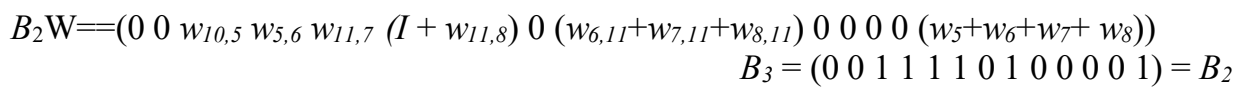

According to the results obtained, we can conclude that the change of the influence on the target indicator depends on the concepts, which were influenced.

The combination of neutrosophic cognitive modeling with models of neuro-fuzzy inference provides the opportunity to carry out a deep analysis of semi-structured problems. Another advantage is an ability to enter additional primary data based on the expert assessment, which in certain cases may lead to an increase in the reliability of the conclusions to a decision-maker.

\section{Conclusion}

1. Based on the synthesis of interdisciplinary research strategies the authors have presented a structural and functional scheme of an intellectual multilevel system of analysis and forecast of scientific and technological, innovative research projects, innovative facilities, and areas. Research strategies included expert foresight methods, which represent a system of peer review of strategic prospects of innovative development, identification of medium and long-term technological breakthroughs capable to have the greatest impact on the economy and society, fuzzy inference models, identification of parameters of neural 
network technologies, and fuzzy cognitive models. The core of the system is formed by the designed multicriteria models of adaptive neuro-fuzzy inference and fuzzy cognitive analysis aimed to solve a number of problems and forecast evaluation scenarios of multidimensional attribute of objects and processes. The system developed has the following distinctive features:

- distributed iterative information system that operates in a real time-mode;

- possibility to make a foresight forecast of the future;

- adaptability to goals, tasks and problems because of the possibility to adjust to the neuro-fuzzy and cognitive model;

- logical transparency, which allows you to accumulate knowledge in the sphere of analytical and forecast problems of innovative development;

- universality, which means the possibility to apply various innovative studies, projects and objects (including enterprises, industries, territories (cities, regions, innovation complexes) for analyzing and forecasting the development.

2. The use of fuzzy cognitive maps as a tool for modeling semi-structured systems allows representing the system under analysis clearly and flexibly, as well as to interpret causal relationships between concepts.

3. Combining neutrosophic cognitive modeling with the results of neuro-fuzzy conclusion resulted in a new intelligent foresight tool, which provides logical transparency of the forecasting studies. It gives the opportunity for deep and quick analysis of scientific, technological, and innovation development taking into account many signs, their significance, mutual influence, and contingency with multiplier effects. It also allows carrying out a situational analysis depending on the target factor. On the other hand, the methods give the possibility to introduce additional expert data, which in general case can increase the reliability of the conclusions.

4. The results obtained have the practical value, which includes the following main factors. They allow improving the quality of forecasts of the most important areas of scientific and technological development of the state objects and territories. The results also allow identifying factors determining the prospects for economic modernization and the enhancement of the effectiveness of scientific and technical policy aimed at outstripping the development of basic production and transition to a new technological order.

The correspondence of the results obtained with the world level of research makes it possible to use them in the economic, scientific, technological, and social spheres with the purpose of scientifically substantiated support of decision-making procedures. Prospects for further research are related to the development of algorithms and methods for parametric and structural identification of the models constructed.

\section{Acknowledgements}

The article is prepared within the framework of Competitiveness Enhancement Programme of National Research Tomsk State University and grant of the Russian Foundation for Basic Research No 16-29-12858.

The authors are grateful to Tatiana B. Rumyantseva from the National Research Tomsk State University for her assistance in preparing the paper.

\section{References}

[1] S.K. Glazyev, Theory of long-term technical and economic development (FIZMATLIT, Moscow, 1993) 
[2] T.V. Abramova, E.V.Vaganova, S.V. Gorbachev, M.V. Gribovskiy, V.I. Syryamkin, M.V. Syryamkin, T.V. Yakubovskaya, Cognitive systems for monitoring and forecasting the state's scientific and technological development (Publishing house of Tomsk State University, 2012)

[3] J. Forrester, World Dynamics (Science, Moscow, 1978)

[4] S.V. Gorbachev, T.V. Abramova, Telecom. 7, 43 (2014)

[5] R. Popper, Methodology: Common Foresight Practices \& Tools (Edward Elgar, 2007)

[6] S.V. Gorbachev, V.I. Syryamkin, Neuro-fuzzy methods in intelligent systems for processing and analyzing multidimensional information (Tomsk State University, 2014)

[7] D. Rutkovskaya, M. Pilinsky, L. Rutkovsky, Neural networks, genetic algorithms and fuzzy systems (Moscow, 2006)

[8] S.V. Gorbachev, Fazzification of expert linguistic data in the ordinal scale (Kursk, 2016)

[9] S.V. Gorbachev, Designing a Hybrid Neural-Fuzzy System Based on Soft Computing (Tomsk State University, 2014)

[10]A.A. Kulinich, Methodology of cognitive modeling of complex ill-defined situations (ISP RAS, Moscow,2003)

[11]L.A. Ginis, Scient. Rev. 8, 219 (2015) 